\title{
Magnetoliposomes: Liposomal Contrast Agents
}

\author{
D. FrAsCIONE ${ }^{1}$, M. SABA-LEPEK ${ }^{1}$, C. DIWOKY ${ }^{2}$, P. OPRIESSNIG ${ }^{2}$, \\ R. Stollberger ${ }^{2}$, G. Almer ${ }^{3}$, H. MANGge ${ }^{3}$, R. Prassl ${ }^{1}$ \\ ${ }_{2}^{1}$ Institute of Biophysics and Nanosystems Research, Austrian Academy of Sciences, Graz, 8042, Austria \\ ${ }^{2}$ Institute of Medical Engineering, University of Technology, Graz, 8010, Austria \\ ${ }^{3}$ Medical University, Graz, 8010, Austria \\ E-mail: daniela.frascione@oeaw.ac.at (D. Frascione)
}

Sci Pharm. 2010; 78: 569

doi:10.3797/scipharm.cespt.8.LNM01

Magnetoliposomes (MLs) are phospholipid vesicles encapsulating magnetic nanoparticles utilised as contrast agents for targeted molecular Magnetic Resonance Imaging (MRI). There are different methods of preparation and characterization of liposomes containing ultra small paramagnetic iron oxide ions (USPIOs) i. e. lipid film hydration method [1] or reverse phase evaporation [2]. In this work as magnetic core we have used commercially available dextran coated iron particles (Molday-ION, purchased by Bio-Pal, USA), with a colloidal size of $30 \mathrm{~nm}$. Liposomes were prepared by thin lipid film hydration method: a predetermined mixture of lipids made of phosphatidylcholine, with or without cholesterol and small amounts of PEG-ylated lipids (which sterically stabilize the liposome reducing their usual rapid uptake from the immune system) was dissolved in organic solvent and evaporated to dryness. Then the dry lipid film was hydrated with a buffered aqueous solution of different concentration of dextran magnetite. The resulting MLs, which were heterogeneous in their size, were centrifuged suddenly after the hydration step to remove excess dextran magnetite; some MLs were sonicated before centrifugation with an Ultra Sonicator (50 min, 130 Watt) to obtain a homogeneous particle size. Physical characterization was performed by dynamic light scattering (DLS) to determine the mean particles diameter and the size distribution. These data were compared to morphological images obtained by transmission electron microscopy (TEM). Chemically, the absolute amount of $\mathrm{Fe}$ in MLs was determined by a colometric test using potassium thiocyanate (KSCN) as reagent. MRI measurements were performed in vitro in agarose gel phantoms to evaluate the intensity of the contrast agent on T1 and T2 relaxation time. In vivo, the biodistribution and the cleareance of the particles over time were studied in mice. MLs have shown a high negative and positive enhancement in MRI and due to this strong contrast effect they will be modified for non invasive diagnosis of atherosclerotic plaques by targeted molecular imaging.

[1] Leszcyznska D, Leszcyznska J, Babincova M, Babinec P. Magnetoliposome composition for targeted treatment of biological tissue and associated methods. Patent Application Publication. Pub.No.: US 2003/0211045 A1. Pub.Date: Nov. 13, 2003.

[2] Viroonchatapan E, Ueno M, Sato H, Adachi I, Nagae H, Tazawa K, Horikoshi I. Preparation and Characterization of Dextran Magnetite-Incorporated Thermosensitive Liposomes. Pharm Res. 1995; 12: 1176-1183. doi:10.1023/A:1016216011016 